\title{
ON THE STABILITY OF SOLITARY KINETIC ALFVÉN WAVES
}

\author{
J.P. SHEERIN \\ Department of Physics, University of Michigan, Ann Arbor, MI 48109, USA \\ and
}

R.S.B. ONG

Department of Aerospace Engineering, University of Michigan, Ann Arbor, MI 48109, USA

Received 21 June 1979

\begin{abstract}
The stability of the solitary kinetic Alfvén wave is investigated. In the one-dimensional fully nonlinear case the wave is shown to be stable against perturbations in the amplitudes.
\end{abstract}

Solitary Alfvén waves have been employed in the description of large amplitude magnetic structures in space- and astro-physics. In an isotropic plasma it is well known that such localized waves are subject to a tendency of collapse in two or more dimensions [1]. However, the presence of a large ambient magnetic field, and hence anisotropy, may serve to stabilize the wave against collapsing. Therefore, it is of interest to study solitary waves in a strongly magnetized plasma.

Hasegawa and Mima have recently discussed the structure of localized finite amplitude Alfvén waves based on kinetic theory [2]. The ratio of the thermal to magnetic pressure, $\beta$, is assumed small, i.e.

$m_{\mathrm{e}} / m_{\mathrm{i}} \ll \beta \ll 1$.

This low $\beta$ approximation allows us to use two scalar potentials to describe the electric fields, as it is equivalent to a shear wave approximation. The component in the direction of the ambient magnetic field is described by the potential $\psi$, and the perpendicular component by the potential $\phi$. The longitudinal current is primarily due to electrons. For time scales of the order of the inverse ion Larmor frequency the electrons are able to thermalize along the ambient magnetic field $\boldsymbol{B}_{0}$ under the influence of $\psi$, i.e.

$n_{\mathrm{e}}=n_{0} \exp \left(e \psi / T_{\mathrm{e}}\right)$.

On the other hand the ions provide a polarization drift transverse to $\boldsymbol{B}_{0}$,

$V_{\mathrm{i} x}=\left(-e / m_{\mathrm{i}} \Omega_{\mathrm{i}}^{2}\right)\left(\partial^{2} \phi / \partial x \partial t\right)$.

Scaling the transverse coordinate to $\rho_{\mathrm{s}}=\left(T_{\mathrm{e}} / m_{\mathrm{j}}\right)^{1 / 2} /$ $\Omega_{\mathrm{i}}$, the longitudinal coordinate to $c / \omega_{\mathrm{pi}}$, and the potentials to $T_{\mathrm{e}} / e$, a "one-dimensional" nonlinear wave equation for the density is derived [2],

$K_{x}^{2} K_{z}^{2} \bar{n}(\mathrm{~d} / \mathrm{d} \eta)\left[\bar{n}^{-1}(\mathrm{~d} \bar{n} / \mathrm{d} \eta)\right]$

$$
+\left(K_{z}^{2}-\bar{n}\right)(1-\bar{n})=0,
$$

where $\eta \equiv K_{x} \xi+K_{z} \zeta-\tau, \xi=x / \rho_{\mathrm{s}}, \zeta=z \omega_{\mathrm{pi}} / c, \tau=\Omega_{\mathrm{i}} t$, $\bar{n}=n / n_{0}$. The nonlinear wave equation (1) may be written in the form

$\mathrm{d}^{2} \bar{n} / \mathrm{d} \eta^{2}+V^{\prime}(\bar{n})=0 ，$

where

$$
\begin{aligned}
& V^{\prime}(\bar{n})=\mathrm{d} V(\bar{n}) / \mathrm{d} \bar{n}=\left(-2 / K_{x}^{2} K_{z}^{2}\right)\left\{\frac{1}{2}(1-\bar{n})\left(K_{z}^{2}+3 \bar{n}\right)\right. \\
& \left.\quad+\left(1+K_{z}^{2}\right) \bar{n} \ln \bar{n}\right\} .
\end{aligned}
$$

Eq. (2) is in the form of the nonlinear Klein-Gordon equation:

$\left(\partial^{2} / \partial t^{2}-\nabla^{2}\right) \bar{n}=V^{\prime}(\bar{n})$

and it may be investigated by means of a method described by Whitham [3]. First, eq. (2) is integrated once to yield 
$\frac{1}{2}(\mathrm{~d} \bar{n} / \mathrm{d} \eta)^{2}+V\left(\bar{n} ; K_{z}^{2}\right)=0 ，$

where

$$
\begin{aligned}
& V\left(\bar{n}, K_{z}^{2}\right)=\left(-\bar{n}^{2} / K_{x}^{2} K_{z}^{2}\right)\left\{(1-\bar{n})\left(K_{z}^{2}+n\right)\right. \\
& \left.\quad+\left(1+K_{z}^{2}\right) \bar{n} \ln \bar{n}\right\} .
\end{aligned}
$$

The formal solution to eq. (4) is given by

$\eta=2^{-1 / 2} \int \mathrm{d} \bar{n}\{A-V(\bar{n})\}^{-1 / 2}$,

where a constant $A$ is included for completeness. We have $1 \leqslant \bar{n} \leqslant \bar{n}_{\max }$ and $A-V(\bar{n}) \geqslant 0$. The lagrangian for the system is given by

$L=\frac{1}{2}(\mathrm{~d} \bar{n} / \mathrm{d} \eta)^{2}-V(\bar{n})$,

where $V(\bar{n})$ is given by eq. (5). We may now perform the following hamiltonian transformation

$\Pi=\partial L / \partial \bar{n}_{\eta}=\bar{n}_{\eta}$,

where $\bar{n}_{\eta}=\partial \bar{n} / \partial \eta$. The hamiltonian is given by

$H=\bar{n}_{\eta} \Pi-L=\frac{1}{2} \Pi^{2}+V(\bar{n})$.

The integral $H=A$ is now solved for $\Pi$. From eq. (9) we obtain

$\Pi=2^{1 / 2}\{A-V(\bar{n})\}^{1 / 2}$.

The averaged lagrangian for a cycle is given by

$$
\begin{aligned}
\mathcal{L} & =(2 \pi)^{-1} \oint \Pi \mathrm{d} \bar{n}-A \\
& =\left(2^{1 / 2} \pi\right)^{-1} \oint\{A-V(\bar{n})\}^{1 / 2} \mathrm{~d} \bar{n}-A .
\end{aligned}
$$

Eq. (11) is of the form $\mathcal{L}=F(A)-A$, with

$F(A) \equiv(2 \pi)^{-1} \oint 2^{1 / 2}\{A-V(\bar{n})\}^{1 / 2} \mathrm{~d} \bar{n}$.

The characteristic equation is

$\mathrm{d} x / \mathrm{d} t=(1 \pm U \sqrt{h}) /(U \pm \sqrt{h})$,

where $h=-F F^{\prime \prime} /\left(F^{\prime}\right)^{2}$ and $U=\omega / k$. If $h<0$, the characteristic velocity is complex. Since small amplitude wave perturbations vary as $F \approx \exp \{\mathrm{i} \alpha(x-U t)\}$, a complex characteristic velocity implies an exponential growth in time. As both $F$ and $\left(F^{\prime}\right)^{2}$ are positive, so we need $F^{\prime \prime}<0$ for stability. Now,

$$
\begin{aligned}
F^{\prime \prime} & =-(8 \pi)^{-1} \oint 2^{1 / 2}\left\{A+\left(\bar{n} / K_{X}^{2} K_{z}^{2}\right)\left[(1-\bar{n})\left(K_{z}^{2}+\bar{n}\right)\right.\right. \\
& \left.\left.+\left(1+K_{Z}^{2}\right)(\bar{n} \ln \bar{n})\right]\right\}^{-3 / 2} \mathrm{~d} \bar{n} .
\end{aligned}
$$

For $1 \leqslant \bar{n} \leqslant \bar{n}_{\text {max }}$ the logarithmic term is positive and dominant. Then for $A \geqslant 0$ the integral in eq. (14) is positive and so $F^{\prime \prime}(A)$ is negative, satisfying a necessary condition for stability. Thus in the one-dimensional fully nonlinear case the solitary kinetic Alfven wave is stable against perturbations in the amplitude.

This work was supported by Grant AFOSR-76-2904 and the Michigan Memorial Phoenix Project.

\section{References}

[1] J.P. Gibbons et al., J. Plasma Phys. 17 (1977) 153.

[2] A. Hasegawa and K. Mima, Phys. Rev, Lett. 37 (1976) 690.

[3] G.B. Whitham, Linear and nonlinear waves (Wiley-Interscience, New York, 1974). 